УДК 519.21

DOI https://doi.org/10.32838/2663-5941/2020.4/11

Дубко В.О.

Таврійський національний університет імені В.І. Вернадського

\title{
МОДЕЛЮВАННЯ СИСТЕМ 3 ВИПАДКОВОЮ СТРУКТУРОЮ ЗА ДОПОМОГОЮ ІНДИКАТОРНИХ ВИПАДКОВИХ ПРОЦЕСІВ
}

У иій статті наведені наші результати, пов'язані з використанням інваріантної властивості індикаторного випадкового прочесу під час моделювання, що має фізичну інтерпретацію випадкових процесів. Розглянуто метод конструктивної побудови, алгоритму моделювання систем зі змінною структурою. Підкреслено, що рівняння з випадковими коефіиієнтами використовуються під час моделювання динамічних систем, які можуть адаптуватися до зовнішніх змін, у тому числі і випадкових, з метою збереження важливих для існування системи показників. Ці зміни в структурі системи можуть бути неперервними або дискретними. Якщзо зміни зовнішніх впливів випадкові, то перехід з одного стану в інший можна намагатися моделювати, спираючись на теорію систем масового обслуговування. Але для опису динаміки реалізачій иих станів необхідні стохастичні рівняння. Як робоче поняття вводиться визначення «індикаторний випадковий прочес». Індикаторним випадковим проиесом названа стрибкоподібна випадкова величина, яка може приймати на розділених між собою інтервалах часу тільки два значення: 0 або 1. Показано, що індикаторний випадковий процес є незмінним (інваріантним) у разі зведення до довільного додатного ступеня. Як приклад застосування індикаторного випадкового процесу знайдена характеристична функція для прочесу, що приймає обмежене число несумісних значень.

Скориставщись поданням індикаторного випадкового процесу як функиії від процесу Пуассона, побудовані та досліджені рівняння для динамічних процесів у середовищі із властивостями, що стрибкоподібно змінюються. 3 урахуванням додаткових зовнішніх збурень приклади таких рівнянь побудовані з використанням рівнянь Іто.

Для демонстращії і більш повного дослідження можливостей алгоритму повністю досліджені динамічні моделі для процесу дифузї в середовищі з центрами тимчасової затримки, процесів дифузї у разі переходів стрибком з одного підпростору в інший і назад. Для цих моделей побудовані рівняння для характеристичних функиій. Знайдено точні розв'язки ичих рівнянь та вказано на взаємозв'язок між иими розв'язками.

Як приклад застосування методу індикаторних функиій побудована характеристична функиія для моделі Каца. Показано, щьо у разі залежності індикаторного випадкового процесу від процесу Пуассона рівняння для характеристичної функиї відповідає телеграфному рівнянню. Цей результат збігається з результатом у роботі Кайа.

Ключові слова: характеристична функиія, випадковий прочес, випадкова структура, рівняння Іто, проиес Пуассона.

Постановка проблеми. Рівняння зі змінною структурою використовуються під час моделювання динамічних систем, що можуть адаптуватись до зовнішніх змін, у тому числі і випадкових, 3 метою збереження життєво визначальних для існування системи показників [1].

Ці зміни у структурі системи можуть бути неперервними, дискретними та змішаного типу. Якщо зміни зовнішніх впливів дискретно-випадкові, то одним зі шляхів моделювання переходів 3 одного стану системи в інший є використання методів теорії систем масового обслуговування. Але для опису динаміки реалізацій цих станів потрібно використовувати стохастичні рівняння.

У цій статті ми розглянемо метод конструктивної побудови (алгоритму) моделей таких систем та продемонструємо його можливості на моделях, що мають фізичну інтерпретацію.
Виклад основного матеріалу досліджень.

Надалі під час побудови рівнянь нам знадобляться деякі робочі поняття та допоміжні твердження.

Індикаторним випадковим прочесом (IBП) назвемо стрибкоподібний випадковий процес $\chi(t)$, який може приймати лише два значення: 1 або 0.

Нехай $\quad p(\chi(t)=1)=p(t) .3$ означення $\chi(t)$ випливає, що

$$
\begin{gathered}
\forall t, \chi^{\alpha}(t)=\chi(t), \forall \alpha>0, \\
p(\chi(t)=1)=M[\chi(t)], \\
p(\chi(t)=0)=1-M[\chi(t)]
\end{gathered}
$$

Лема 1. $(1-\chi(t))^{m}=(1-\chi(t))$ для будь-якого додатного $m$. 
Доведення. Врахувавши (1), переконуємось, що

$$
\begin{aligned}
& (1-\chi(t))^{m}=\left(1-\sum_{l=1}^{m}(-1)^{l} C_{n}^{l} \chi(t)^{l}\right)= \\
& =\left(1-\chi(t) \sum_{l=1}^{m}(-1)^{l} C_{n}^{l}\right)=(1-\chi(t))
\end{aligned}
$$

Лема 2. Нехай $\chi_{j}(t), j=\overline{1, n-1}$ набір незалежних процесів. Тоді набір процесів

$$
\begin{gathered}
z_{k}=\chi_{k}(t) \prod_{j=1}^{k-1}\left(1-\chi_{j}(t)\right), \\
z_{l}=\chi_{l}(t) \prod_{j=1}^{l-1}\left(1-\chi_{j}(t)\right), \\
z_{1}=\chi_{k}(t), \quad z_{n}=\prod_{j=1}^{n-1}\left(1-\chi_{j}(t)\right), \forall k, l
\end{gathered}
$$

утворює повну групу несумісних процесів.

Доведення. Спираючись на Лему1, знаходимо

$$
\begin{aligned}
& z_{k} z_{l}=\chi_{k}(t)\left(1-\chi_{k}(t)\right) \chi_{l}(t) \prod_{j=1, j \neq k}^{k}\left(1-\chi_{j}(t)=\right. \\
& =\left[\chi_{k}(t)\left(1-\chi_{k}(t)\right)\right] \chi_{l}(t) \prod_{j=1, j \neq k}^{k}\left(1-\chi_{j}(t)\right) \equiv 0
\end{aligned},
$$

так як $\chi_{k}(t)\left(1-\chi_{k}(t)\right)=\chi_{k}(t)-\chi_{k}^{2}(t)=0, \forall k<l$.

$$
\begin{gathered}
z_{1}+\sum_{k=2}^{n-1} z_{k}+z_{n}=\chi_{1}(t)+ \\
+\sum_{k=2}^{n-1} \chi_{k}(t) \prod_{j=1}^{k-1}\left(1-\chi_{j}(t)\right)+\prod_{j=1}^{n-1}\left(1-\chi_{j}(t)\right) \equiv 1
\end{gathered} .
$$

\section{Приклади застосування ІВП.}

Як перший приклад застосування $\chi_{j}(t)$ побудуємо характеристичну функцію (Х.Ф.) $J(t)$ для процесу $y(t)=\sum_{j=1}^{n} y_{j}(t)$, де

$$
\begin{gathered}
y_{1}(t)=\chi_{1}(t) g_{1}(t), \\
y_{k}(t)=\chi_{k}(t) \prod_{j=1}^{k-1}\left(1-\chi_{j}(t)\right) g_{k}(t), . \\
y_{n}(t)=\prod_{j=1}^{n-1}\left(1-\chi_{j}(t)\right) g_{n}(t),
\end{gathered}
$$

$k=\overline{2, n-1} ., g_{k}(t)$ незалежні від $\chi_{j}(t)$ випадкові процеси.

3 урахуванням уведених представлень

$$
\begin{gathered}
y(t)=\chi_{1}(t) g_{1}(t)+ \\
+\sum_{k=2}^{n=1} \chi_{k}(t) \prod_{j=1}^{k-1}\left(1-\chi_{j}(t)\right) g_{k}(t)+ \\
+\prod_{j=1}^{n-1}\left(1-\chi_{j}(t)\right) g_{n}(t) .
\end{gathered}
$$

Через несумісність процесів $z_{k}$ (Лема 2) у довільний момент часу $y(t)$ буде набувати тільки одне з можливих значень $y(t)_{j}$.
Теорема 1. Х.Ф. процесу $y(t)$ така:

$$
\begin{gathered}
J(t)=p_{1}(t) M\left[e^{i \beta g_{1}(t)}\right]+ \\
+\sum_{k=2}^{n-1} M\left[e^{i \beta g_{k}(t)}\right] p_{k}(t) \prod_{j=1}^{k-1}\left[1-p_{j}(t)\right]+ \\
+M\left[e^{i \beta g_{n}(t)}\right] \prod_{j=1}^{n-1}\left[1-p_{j}(t)\right] .
\end{gathered}
$$

Доведення.

$$
\begin{gathered}
J(t)=M[\exp \{i \beta y(t)\}]= \\
=M\left[\operatorname { e x p } \left\{i \beta \left(\chi_{1}(t) g_{1}(t)+\right.\right.\right. \\
+\sum_{k=2}^{n-1} \chi_{k}(t) \prod_{j=1}^{k-1}\left(1-\chi_{j}(t)\right) g_{k}(t)+ \\
\left.\left.\left.+\prod_{j=1}^{n-1}\left(1-\chi_{j}(t)\right) g_{n}(t)\right)\right\}\right]= \\
=M[\exp \{i \beta y(t)\} \times \\
\times \prod_{k=2}^{n-1} \exp \left\{\beta \chi_{k}(t) \prod_{j=1}^{k-1}\left(1-\chi_{j}(t)\right) g_{k}(t)\right\} \times \\
\left.\times \exp \left\{i \beta \prod_{j=1}^{n-1}\left(1-\chi_{j}(t)\right) g_{n}(t)\right\}\right]
\end{gathered}
$$

Розкладемо кожну з експонент у ряд. Врахуємо несумісність процесів $z_{k}$ (Лема 2), властивість (1) та незалежність $g_{k}(t), \chi_{l}(t), \forall k, l$. Після взяття математичного сподівання 3 урахуванням доходимо до твердження Теореми 1.

Скориставшись властивостями $\chi(t)$, можемо побудувати рівняння для динамічних процесів у середовищі зі стрибкоподібною зміною властивостей.

Прикладом можуть бути такі рівняння Іто:

$$
\begin{aligned}
& d x(t)=\chi_{1}(t) a_{1}(x(t) ; t) d t+\chi_{2}(t) B_{1}(x(t) ; t) d w(t)+ \\
& +\left(1-\chi_{1}(t)\right) a_{2}(x(t) ; t) d t+\left(1-\chi_{2}(t)\right) B_{2}(x(t) ; t) d w(t)
\end{aligned}
$$

де $\varnothing_{w}(t)$ - випереджаючий стосовно поточного часу $t$ приріст вінерівського процесу $w(t) \in R^{m}$, $a_{j}(x ; t), x \in R^{n} ; B_{j}(x(t) ; t)$ матриці розмірності $n \times m, j=1,2$.

За допомогою $\chi(t)$ можливо моделювати і рух із переходами з одного підпростору в інший:

$\left\{\begin{array}{l}\left.\frac{d x(t)}{d t}=\chi(t)\left[a_{1}(x(t) ; y(t) ; t)\right) d t+B_{1}(x(t) ; y(t) ; t) d w(t)\right] \\ \left.\frac{d y(t)}{d t}=[1-\chi(t)]\left[a_{2}(x(t) ; y(t) ; t)\right) d t+B_{2}(x(t) ; y(t) ; t) d w(t)\right]\end{array}\right.$, і т. Д..

Якщо

$$
\begin{gathered}
B_{l}(x(t) ; y(t) ; t)=0, \\
a_{1}(x(t) ; y(t) t)=-a_{2}(x(t) ; \\
y(t) t)=-c, c=\text { const }>0,
\end{gathered}
$$

то процес $x(t)+y(t)$ відповідає моделі Каца: рух частинки 3 випадковою зміною напрямку швидкості $c$ [3]. 
Нехай $\chi(t), \chi(0)=1$ - функція від цілочисельної випадкової величини $N(t)$ :

$$
\chi(t)=\chi(N(t))
$$

Якщо

$$
\chi(N(t))=\chi(N(t)+2 k),
$$

коли $k \in$ простору цілих чисел, то $\chi(t)$ назвемо умовно періодичним.

Умовам (1), (3) задовольняс функція:

$$
\chi(t)=\chi(N(t))=\frac{1}{2}(1+\cos [N(t) \pi]) .
$$

Бачимо, що для парних $N(t): \frac{1}{2}(1+\cos [N(t) \pi])=1$. Для непарних $N(t): \frac{1}{2}(1+\cos [N(t) \pi])=0$.

Надалі будемо працювати саме з такими $\chi(t)$.

Якщо $N(t)$ - стаціонарний пуассонівський процес 3 параметром інтенсивності $\lambda$, то $M[\chi(N(t))]=0,5(1+\exp \{-2 \lambda t\})$.

Дифузія з центрами тимчасової випадкової затримки.

Розглянемо приклад системи зі змінною структурою, коли зміни стану системи підпорядковані рівнянню

$$
\begin{gathered}
d y(t)=a(t) d t+\frac{1}{2}(1+\cos [N(t) \pi])[b d w(t)] ; \\
y(0)=0 .
\end{gathered}
$$

Тобто певний час система змінюється за детермінованим законом, а потім на випадковому проміжку часу на неї діють випадкові збурення.

Виконаємо заміну змінних:

$$
y(t)-\int_{0}^{t} a(\tau) d \tau=x(t),
$$

Тепер рівняння (4) набуває вигляду:

$$
d x(t)=\frac{1}{2}(1+\cos [N(t) \pi])[b d w(t)] .
$$

Це рівняння, яке описує дифузію з центрами тимчасової випадкової затримки [2]. Відповідно, характеристична функція для цього процесу

$$
\begin{gathered}
J_{1}(t)=M[f(t)]= \\
=M\left[\exp \left\{-i \beta 2^{-1} \int_{0}^{t}(1+\cos [N(\tau) \pi]) b d w(\tau)\right\}\right] .
\end{gathered}
$$

Дифузія з випадковою зміною напрямку руху. Модель Каца.

Розглянемо одновимірний рух, коли відбувається зміна напрямку

переміщення у випадкові моменти часу [3]:

$$
\left\{\begin{array}{l}
d x^{+}(t)=\chi(t) \mathrm{v} d t \\
d x^{-}(t)=-(1-\chi(t)) \mathrm{v} d t
\end{array}\right.
$$

Оскільки нас цікавить сумарне переміщення $\left[x^{+}(t)+x^{-}(t)\right]=x(t)$, то приходимо до рівняння:

$$
d x(t)=(2 \chi(t)-1) \mathrm{v} d t .
$$

У якості $\chi(t)$ виберемо $\chi(t)=\frac{1}{2}(1+\cos [N(t) \pi])$.

Тоді характеристична функція для процесу $x(t)$ набуває вигляду:

$$
I(t)=M\left[\exp \left\{i \beta \int_{0}^{t} \cos (N(\tau) \pi) c d \tau\right\}\right]
$$

Теорема 2. Х.ф. для процесу $x(t)$, підпорядкованого (7), за умови, що $N(t)$ стаціонарний пуассонівський процес з параметром $\lambda, \epsilon$ розв'язком рівняння:

$$
\begin{gathered}
\frac{d^{2} I(t)}{d t^{2}}+2 \lambda \frac{d I(t)}{d t}+c^{2} \beta^{2} I(t)=0, \\
I(0)=1, \quad \frac{d I(0)}{d t}=i c \beta
\end{gathered}
$$

Доведення.

$$
\begin{gathered}
\frac{d I(t)}{d t}= \\
=M\left[i \beta \cos (N(t) \pi) c \exp \left\{i \beta \int_{0}^{t} \cos (N(\tau) \pi) c d \tau\right\}\right]
\end{gathered}
$$

Скориставшись правилом диференціювання функції $f(y)$, коли $y=N(t)$ - стаціонарний пуассонівський процес:

$$
d f(N(t))=[f(N(t)+1)-f(N(t))] d N(t),
$$

де $d N(t)$ випереджаючий приріст, тобто, який не залежить від попередніх значень $N(t)$. Через властивості розподілу Пуассона з параметром $\lambda$,

$$
M[d N(t)]=\lambda d t, \lambda>0 .
$$


та (9) знаходимо:

$$
d \frac{d I(t)}{d t}=
$$

$=-c^{2} \beta^{2} M\left[\cos ^{2}(N(t) \pi) \exp \left\{i \beta \int_{0}^{t} \cos (N(\tau) \pi) c d \tau\right\}\right] d t+$

$+i \beta c M[(\cos (\{N(t)+1\} \pi)-\cos (N(t) \pi)) \times$

$\left.\times \exp \left\{i \beta \int_{0}^{t} \cos (N(\tau) \pi) c d \tau\right\}\right] \mu d t=$

$=-c^{2} \beta^{2} M\left[\exp \left\{i \beta \int_{0}^{t} \cos (N(\tau) \pi) c d \tau\right\}\right] d t+$

$\left.-i \beta c 2 \lambda M[\cos (N(t) \pi)) \exp \left\{i \beta \int_{0}^{t} \cos (N(\tau) \pi) c d \tau\right\}\right] d t=$

$$
=-c^{2} \beta^{2} I(t) d t-2 \lambda \frac{d I(t)}{d} d t .
$$

Зауваження. Застосувавши обернене перетворення Фур'є до (8), приходимо до телеграфного рівняння для визначення щільності розподілу $\rho(x ; t)$ :

$$
\frac{\partial^{2} \rho(x ; t)}{\partial t^{2}}+2 \lambda \frac{\partial \rho(x ; t)}{\partial t}-c^{2} \frac{\partial^{2}}{\partial x^{2}} \rho(x ; t)=0,
$$

що співпадає з результатами з роботи [3].

Модель дифузії 3 випадковими переходами з одного підпростору в інший. Двовимірний випадок.

Розглянемо таку модель дифузії:

$$
\left\{\begin{array}{l}
d x(t)=\chi(t) b d w(t) \\
d y(t)=(1-\chi(t)) b d w(t)
\end{array} .\right.
$$

Тобто у випадкові моменти часу процес відбувається або у просторі $x(t)$ або $y(t)$.

Як випадковий індикаторний процес виберемо $\chi(t)=2^{-1}(1+\cos N(t))$. Тоді характеристична функція для цієї системи має вигляд:

$$
\begin{aligned}
J(t)= & M\left[\exp \left\{i \alpha \int_{0}^{t} 2^{-1}(1+\cos N(\tau)) b d w(\tau)\right\}+\right. \\
& \left.\left.+i \beta \int_{0}^{t} 2^{-1}(1-\cos N(\tau)) b d w(\tau)\right\}\right]
\end{aligned}
$$

Теорема 3. Якщо $N(t)$ стаціонарний пуассонівський процес 3 параметром $\lambda$, то $J(t) \in$ розв'язком рівняння:

$$
\begin{gathered}
\frac{d^{2} J(t)}{d t^{2}}+2^{-1}\left(4 \lambda+\left[\alpha^{2}+\beta^{2}\right] b^{2}\right) \frac{d J(t)}{d t}+ \\
+2^{-1}\left(\lambda \alpha^{2}+\alpha^{2} \beta^{2} b^{2} 2^{-1}+\lambda \beta^{2}\right) b^{2} M[f(t)]=0 .
\end{gathered}
$$

Доведення. 3 урахуванням незалежності процесів $N(\tau)$ та $w(\tau)$, скориставшись формулою Іто, знаходимо:

$$
\begin{gathered}
J(t)=M\left[\operatorname { e x p } \left\{i \int_{0}^{t} 2^{-1}[(\alpha+\beta)+(\alpha-\beta) \cos (N(\tau)] b d w(\tau)\}=\right.\right. \\
=M\left[\operatorname { e x p } \left\{-\int_{0}^{t} 2^{-3}\left[(\alpha+\beta)+(\alpha-\beta) \cos (N(\tau)]^{2} b^{2} d \tau\right\}=\right.\right. \\
=M\left[\operatorname { e x p } \left\{-\int_{0}^{t} 2^{-3}\left[(\alpha+\beta)^{2}+2\left(\alpha^{2}-\beta^{2}\right) \cos \left(N(\tau)+(\alpha-\beta)^{2}\right] b^{2} d \tau\right\}=\right.\right. \\
=M\left[\exp \left\{-\alpha^{2} \int_{0}^{t} 2^{-2}(1+\cos N(\tau)) b^{2} d \tau\right\}-\right. \\
\left.\left.-\beta^{2} \int_{0}^{t} 2^{-2}(1-\cos N(\tau)) b^{2} d \tau\right\}\right]=M[f(t)]
\end{gathered}
$$

Далі:

$$
\frac{d^{2} J(t)}{d t^{2}}=-2 \lambda \frac{d J(t)}{d t}-2^{-1} \lambda\left(\alpha^{2}+\beta^{2}\right) b^{2} J(t)+
$$

$+M\left[f(t)\left\{\alpha^{4} 2^{-3}\left(1+\cos (N(t)) b^{4}+\beta^{4} 2^{-3}\left(1-\cos (N(t)) b^{4}\right\}\right]\right.\right.$

Скориставшись (10) та (12), отримуємо:

$\frac{d^{2} J(t)}{d t^{2}}=M\left[f(t)\left\{\alpha^{2} 2^{-2} 2 b^{2} \cos N(t)-\beta^{2} 2^{-2} 2 b^{2} \cos (N(t)\}\right] \lambda+\right.$ $+M\left[f(t)\left\{\alpha^{4} 2^{-4}(1+\cos N(t))^{2} b^{4}+\beta^{4} 2^{-4}(1-\cos N(t))^{2} b^{4}\right\}\right]=$ $=\lambda M\left[f(t)\left\{\alpha^{2} 2^{-1}(1+\cos N(t)) b^{2}+\beta^{2} 2^{-1}\left(1-(\cos N(t)) b^{2}\right\}\right]-\right.$

$$
-2^{-1} \lambda\left(\alpha^{2}+\beta^{2}\right) b^{2} J(t)+
$$

$+M\left[f(t)\left\{\alpha^{4} 2^{-3}\left(1+\cos (N(t)) b^{4}+\beta^{4} 2^{-3}\left(1-\cos (N(t)) b^{4}\right\}\right]\right.\right.$.

Далі:

$$
\frac{d^{2} J(t)}{d t^{2}}=-2 \lambda \frac{d J(t)}{d t}-2^{-1} \lambda\left(\alpha^{2}+\beta^{2}\right) b^{2} J(t)+
$$

$+M\left[f(t)\left\{\alpha^{4} 2^{-3}\left(1+\cos (N(t)) b^{4}+\beta^{4} 2^{-3}\left(1-\cos (N(t)) b^{4}\right\}\right]\right.\right.$

Продовжимо перетворення (13).

$$
\begin{gathered}
\frac{d^{2} J(t)}{d t^{2}}= \\
=-2 \lambda \frac{d J(t)}{d t}-2^{-1} \lambda\left(\alpha^{2}+\beta^{2}\right) b^{2} M[f(t)]+ \\
+\alpha^{2} b^{2} 2^{-1} M\left[f ( t ) \left\{\alpha ^ { 2 } 2 ^ { - 2 } \left(1+\cos (N(t)) b^{2}+\right.\right.\right. \\
\left.+\beta^{2} 2^{-2}\left(1-\cos (N(t)) b^{2}\right\}\right]- \\
-\alpha^{2} \beta^{2} b^{2} 2^{-1} M\left[f(t) 2^{-2}\left(1-\cos (N(t)) b^{2}\right]+\right. \\
+M\left[\beta^{4} 2^{-3}\left(1-\cos (N(t)) b^{4}\right\}\right] .
\end{gathered}
$$

Варіант a):

$$
\begin{aligned}
\frac{d^{2} J(t)}{d t^{2}}=- & -\left(2 \lambda+\alpha^{2} b^{2} 2^{-1}\right) \frac{d J(t)}{d t}-2^{-1} \lambda\left(\alpha^{2}+\beta^{2}\right) b^{2} J(t)- \\
-\alpha^{2} \beta^{2} b^{2} 2^{-1} M\left[f(t) 2^{-2}\left(1-\cos (N(t)) b^{2}\right]+\right. & \\
& +M\left[f(t) \beta^{4} 2^{-3}\left(1-\cos (N(t)) b^{4}\right\}\right] .
\end{aligned}
$$


Варіант в):

$$
\begin{aligned}
\frac{d^{2} J(t)}{d t^{2}}=- & \left(2 \lambda+\beta^{2} b^{2} 2^{-1}\right) \frac{d J(t)}{d t}-2^{-1} \lambda\left(\alpha^{2}+\beta^{2}\right) b^{2} J(t)- \\
-\alpha^{2} \beta^{2} b^{2} 2^{-1} M\left[f(t) 2^{-2}\left(1+\cos (N(t)) b^{2}\right]+\right. & \\
& +M\left[f(t) \alpha^{4} 2^{-3}\left(1+\cos (N(t)) b^{4}\right\}\right]
\end{aligned}
$$

Складемо рівняння (14) і (15):

$$
\begin{aligned}
2 \frac{d^{2} J(t)}{d t^{2}} & =-\left(4 \lambda+\left[\alpha^{2}+\beta^{2}\right] b^{2} 2^{-1}\right) \frac{d J(t)}{d t}- \\
-\left(\lambda \alpha^{2}\right. & \left.+\alpha^{2} \beta^{2} b^{2} 2^{-2}+\lambda \beta^{2}\right) b^{2} J(t)-
\end{aligned}
$$

$-M\left[f(t)\left\{\alpha^{4} 2^{-3}\left(1+\cos (N(t)) b^{4}+\beta^{4} 2^{-3}\left(1-\cos (N(t)) b^{4}\right\}\right]\right.\right.$.

Рівняння (13) віднімемо від рівняння (16). Отримаємо:

$$
\begin{gathered}
\frac{d^{2} J(t)}{d t^{2}}+2^{-1}\left(4 \lambda+\left[\alpha^{2}+\beta^{2}\right] b^{2}\right) \frac{d J(t)}{d t}+ \\
+2^{-1}\left(\lambda \alpha^{2}+\alpha^{2} \beta^{2} b^{2} 2^{-1}+\lambda \beta^{2}\right) b^{2} M[f(t)]=0 .
\end{gathered}
$$

Враховуючі введені позначення, приходимо до твердження (11) Теореми 2.

Є можливість знайти одразу розв' язок рівняння (11). Його побудова буде громіздкою. Ми відшукаємо розв'язок для більш простої моделі, а потім вкажемо, як перейти до явного вигляду розв'язку рівняння (11).

Характеристична функція для моделі дифузії 3 центрами затримки

Повернемось до рівняння (5). Характеристична функція $J_{1}(t)$ для процесу $x(t) \in R^{1}$ така (6):

$$
\begin{gathered}
J_{1}(t)=M[f(t)]= \\
=M\left[\exp \left\{-i \beta \frac{1}{2} \int_{0}^{t}(1+\cos [N(\tau) \pi]) b d w(\tau)\right\}\right]
\end{gathered}
$$

Рівняння для визначення $J_{1}(t)$ повинно співпадати з (11), якщо покласти у ньому $\alpha=0$. Формально це відповідає таким замінам коефіцієнтів y (11):

$$
\begin{gathered}
2^{-1}\left(4 \lambda+\left[\alpha^{2}+\beta^{2}\right] b^{2}\right) \Rightarrow 2^{-1}\left(4 \lambda+\beta^{2} b^{2}\right), \\
2^{-1}\left(\lambda \alpha^{2}+\alpha^{2} \beta^{2} b^{2} 2^{-1}+\lambda \beta^{2}\right) b^{2} \Rightarrow 2^{-1} \lambda \beta^{2} b^{2} .
\end{gathered}
$$

Будемо шукати розв'язок більш простого за виглядом рівняння (5), що відповідає моделі дифузії з центрами затримки:

$$
\frac{d^{2}}{d t^{2}} J_{1}(t)+\frac{1}{2}\left(\beta^{2} b^{2}+4 \lambda\right) \frac{d}{d t} J_{1}(t)+\frac{1}{2} \lambda \beta^{2} b^{2} J_{1}(t)=0
$$

Оскільки

$$
\begin{gathered}
\frac{1}{4}\left(\beta^{2} b^{2}+4 \lambda\right)^{2}-4 \frac{\lambda}{2} \beta^{2} b^{2}= \\
=\frac{1}{4} \beta^{4} b^{4}+2 \beta^{2} b^{2} \lambda+4 \lambda^{2}-2 \beta^{2} b^{2} \lambda=, \\
=\frac{1}{4} \beta^{4} b^{4}+4 \lambda^{2}>0, \forall \lambda>0 .
\end{gathered}
$$

то розв'язок ([4, с. 375, ф-ла 235(a)]) буде таким:

$$
\begin{gathered}
J_{1}(t)=C_{1} \exp \left\{-t \frac{1}{2}\left(\beta^{2} b^{2}+4 \lambda\right)+t \sqrt{\frac{1}{4} \beta^{4} b^{4}+4 \lambda^{2}}\right\}+ \\
+C_{2} \exp \left\{-t \frac{1}{2}\left(\beta^{2} b^{2}+4 \lambda\right)-t \sqrt{\frac{1}{4} \beta^{4} b^{4}+4 \lambda^{2}}\right\} .
\end{gathered}
$$

Оскільки

$$
\frac{1}{4}\left(\beta^{2} b^{2}+4 \lambda\right)^{2}>\frac{1}{4} \beta^{4} b^{4}+4 \lambda^{2},
$$

то перші і другі доданки зменшуються зі зростанням $t$, і тому

$$
\lim _{t \rightarrow \infty} J_{1}(t)=0 .
$$

Враховуючи початкові умови

$$
\left.\frac{d}{d t} J_{1}(t)\right|_{t=0}=-\frac{1}{2} \beta^{2} b^{2},\left.J_{1}(t)\right|_{t=0}=1,
$$

отримаємо рівняння для визначення сталих:

$$
\begin{gathered}
C_{1}+C_{2}=1 \\
\frac{1}{2} \beta^{2} b^{2}=C_{1}\left[\frac{1}{2}\left(\beta^{2} b^{2}+4 \lambda\right)+\sqrt{\frac{1}{4} \beta^{4} b^{4}+4 \lambda^{2}}\right]+ \\
+C_{2}\left[\frac{1}{2}\left(\beta^{2} b^{2}+4 \lambda\right)-\sqrt{\left.\frac{1}{4} \beta^{4} b^{4}+4 \lambda^{2}\right]}\right. \\
\frac{1}{2} \beta^{2} b^{2}=\left(1-C_{2}\right)\left[\frac{1}{2}\left(\beta^{2} b^{2}+4 \lambda\right)+\sqrt{\frac{1}{4} \beta^{4} b^{4}+4 \lambda^{2}}\right]+ \\
+C_{2}\left[\frac{1}{2}\left(\beta^{2} b^{2}+4 \lambda\right)-\sqrt{\frac{1}{4} \beta^{4} b^{4}+4 \lambda^{2}}\right] \\
2 \lambda=C_{2} \sqrt{\frac{1}{4} \beta^{4} b^{4}+4 \lambda^{2}} .
\end{gathered}
$$

Розв'язавши цю систему рівнянь, встановлюємо:

$$
\begin{gathered}
C_{2}=2 \lambda / \sqrt{\frac{1}{4} \beta^{4} b^{4}+4 \lambda^{2}}=\left(\frac{1}{16} \lambda^{-2} \beta^{4} b^{4}+1\right)^{-0,5}, \\
C_{1}=1-\left(\frac{1}{16} \lambda^{-2} \beta^{4} b^{4}+1\right)^{-0,5} .
\end{gathered}
$$


Таким чином, розв’язок набуває вигляду:

$$
\begin{gathered}
J_{1}(t)=\exp \left\{-t \frac{1}{2}\left(\beta^{2} b^{2}+4 \lambda\right)+t \sqrt{\frac{1}{4} \beta^{4} b^{4}+4 \lambda^{2}}\right\}+ \\
+\left(\frac{1}{16} \lambda^{-2} \beta^{4} b^{4}+1\right)^{-0,5}\left[\exp \left\{-t \frac{1}{2}\left(\beta^{2} b^{2}+4 \lambda\right)-t \sqrt{\frac{1}{4} \beta^{4} b^{4}+4 \lambda^{2}}\right\}-\right. \\
\left.-\exp \left\{-t \frac{1}{2}\left(\beta^{2} b^{2}+4 \lambda\right)+t \sqrt{\frac{1}{4} \beta^{4} b^{4}+4 \lambda^{2}}\right\}\right]
\end{gathered}
$$

Оскільки

$$
+\left(\frac{1}{16} \lambda^{-2} \beta^{4} b^{4}+1\right)^{-0,5}<1,
$$

то $J(t)>0, \forall t \geq 0$
Скориставшись оберненими замінами коефіцієнтів на основі співвідношення (17), отримаємо і розв'язок (11).

Тепер, враховуючи властивість - зв'язок між характеристичним функціями та моментами, можемо знайти довільні моменти для розглянутих процесів.

Висновки. Як бачимо, метод індикаторних процесів із використанням методу характеристичних функцій дає змогу розглянути як відомі моделі раніше, так і нові, що мають фізичну трактовку.

Відзначимо, що якщо задана повна група несумісних процесів, то встановлюється, що $є$ набір незалежних випадкових індикаторних функцій, на основі яких можна побудувати повну групу, 3 розподілом якої буде співпадати розподіл заданої групи несумісних подій.

\section{Список літератури:}

1. Дубко В.А. Интегральные инварианты уравнений Ито и их связь с некоторыми задачами теории случайных процессов. Док. НАН Украиньл, 2002, № 1. С. 24-29.

2. Дубко В.О. Моделювання розповсюдження домішок у середовищі з центрами затримки за допомогою індикаторних функцій. Аграрна наука та освіта в ХХІ столітті: проблеми, перспективи та інновації : 3б. наукових праць. Ніжин, 2018. С. 313-317.

3. Кац М. Несколько вероятностных задач физики и математики. Москва : Наука, 1967. 176 с.

4. Камке Э. Справочник по обыкновенным дифференциальным уравнениям. Москва : Наука, 1976.576 с.

\section{Doobko V.A. SIMULATION OF SYSTEMS WITH A RANDOM STRUCTURE USING INDICATORS OF RANDOM PROCESSES}

This article presents our results related to the use of the invariance property of an indicator stochastic process when modeling stochastic processes that have a physical interpretation. A method of constructive construction, an algorithm for modeling systems with a changing structure is considered. It is emphasized that equations with random coefficients are used in modeling dynamic systems that can adapt to external changes, including random ones, in order to preserve indicators important for the existence of a system. These changes in the structure of the system can be continuous or discrete. If changes in external influences are random, then the transition from one state to another, you can try to simulate, relying on the theory of queuing systems. But to describe the dynamics of realizations of these states, stochastic equations are needed. As a working concept, a definition is introduced: indicator random process. An indicator random process is a jump-like random variable that can take only two values at intervals of time separated from each other: 0 or 1.

It is shown that the indicator random process is unchanged (invariant) when raised to an arbitrary positive power. As an example of the application of an indicator random process, a characteristic function is found for a process that takes a finite set of incompatible values.

Using the representation of an indicator random process as a function of a Poisson process, equations for dynamic processes in a medium with abruptly changing properties are constructed and investigated. Taking into account additional external disturbances, examples of such equations are constructed using the Itô equations.

For a demonstration and a more complete study of the capabilities of the algorithm, dynamic models for the diffusion process in a medium with centers of time delay, diffusion processes during jump transitions from one subspace to another and back are fully investigated. For these models, equations for the characteristic functions are constructed. Exact solutions of these equations are found and the relationship between these solutions is established.

As an example of the application of the method of indicator functions, the characteristic function for the Katz model is constructed. It is shown that when the indicator random process depends on the Poisson process, the equation for the characteristic function corresponds to the telegraph equation. This result coincides with the result in Katz's work.

Key words: characteristic function, random process, random structure, Ito's equation, Poisson process. 